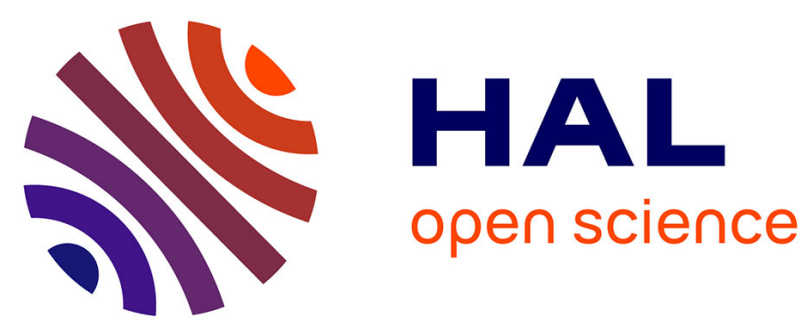

\title{
Reevaluation of the diametral compression test for tablets using the flattened disc geometry
}

Vincent Mazel, Sandra Guerard, Benjamin Croquelois, Jean-Benoit Kopp, Jérémie Girardot, Harona Diarra, Virginie Busignies, Pierre Tchoreloff

\section{- To cite this version:}

Vincent Mazel, Sandra Guerard, Benjamin Croquelois, Jean-Benoit Kopp, Jérémie Girardot, et al.. Reevaluation of the diametral compression test for tablets using the flattened disc geometry. International Journal of Pharmaceutics, 2016, 513 (1-2), pp.669-677. 10.1016/j.ijpharm.2016.09.088 . hal-01378705

\section{HAL Id: hal-01378705 \\ https://hal.science/hal-01378705}

Submitted on 10 Oct 2016

HAL is a multi-disciplinary open access archive for the deposit and dissemination of scientific research documents, whether they are published or not. The documents may come from teaching and research institutions in France or abroad, or from public or private research centers.
L'archive ouverte pluridisciplinaire HAL, est destinée au dépôt et à la diffusion de documents scientifiques de niveau recherche, publiés ou non, émanant des établissements d'enseignement et de recherche français ou étrangers, des laboratoires publics ou privés. 


\title{
Reevaluation of the diametral compression test for tablets using the flattened disc geometry
}

\author{
V. Mazel ${ }^{\mathrm{a}, *}$, S. Guerard ${ }^{\mathrm{b}}$, B. Croquelois ${ }^{\mathrm{a}}$, J.B. Kopp ${ }^{\mathrm{b}}$, J. Girardot $^{\mathrm{b}}$, H. Diarra $^{\mathrm{c}}$, V. Busignies $^{\mathrm{a}}$, \\ P. Tchoreloff ${ }^{\mathrm{a}}$ \\ a Univ. Bordeaux, CNRS, Bordeaux INP, Arts et Métiers ParisTech, I2M, UMR 5295, F-33000 Bordeaux, France \\ ${ }^{\mathrm{b}}$ Arts et Métiers ParisTech, Univ. Bordeaux, Bordeaux INP, I2M, UMR 5295, F-33000 Bordeaux, France

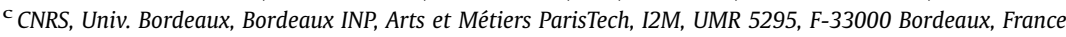

Keywords:

Tablet

Diametral compression

Tensile strength

Compression

Numerical simulation

\begin{abstract}
A B S T R A C T
Mechanical strength is an important critical quality attribute for tablets. It is classically measured, in the pharmaceutical field, using the diametral compression test. Nevertheless, due to small contact area between the tablet and the platens, some authors suggested that during the test, the failure could occur in tension away from the center which would invalidate the test and the calculation of the tensile strength. In this study, the flattened disc geometry was used as an alternative to avoid contact problems. The diametral compression on both flattened and standard geometries was first studied using finite element method (FEM) simulation. It was found that, for the flattened geometry, both maximum tensile strain and stress were located at the center of the tablet, which was not the case for the standard geometry. Experimental observations using digital image correlation (DIC) confirmed the numerical results. The experimental tensile strength obtained using both geometries were compared and it was found that the standard geometry always gave lower tensile strength than the flattened geometry. Finally, high-speed video capture of the test made it possible to detect that for the standard geometry the crack initiation was always away from the center of the tablet.
\end{abstract}

\section{Introduction}

Mechanical strength is an important critical quality attribute for tablets. As such, especially in the development of a Quality by Design approach, a precise and reliable quantification is required. For pharmaceutical tablets, the main technique to study the mechanical strength of the tablet is the diametral compression test (also known as Brazilian test)(EUP, 2016; USP, 2013). This test was developed during the 40's to study the mechanical strength of concrete cylinders (Carneiro, 1943; Fairbairn and Ulm, 2002). It measures the tensile strength in an indirect manner when direct tensile tests are difficult to perform due to the mechanical properties of the tested material. Firstly used for concrete or rocks, it was introduced, during the 60's for the characterization of pharmaceutical tablets (Fell and Newton, 1968, 1970). The cylindrical shape is indeed easy to obtain when performing die compression and the test is thus well suited for tablets.

\footnotetext{
* Corresponding author at: I2M, Univ. Bordeaux, 146 rue Léo Saignat, F-33000, Bordeaux, France.

E-mail address: vincent.mazel@u-bordeaux.fr (V. Mazel).
}

Nevertheless, as mentioned before, the diametral compression test is an indirect test. A cylindrical sample is submitted to a compressive force along its diameter by diametral compression between two flat platens. This promotes the development of tensile stresses at the center of the compact. These tensile stresses are supposed to cause the failure. Then, by supposing an elastic behavior of the compact and by using the elastic theory, in a 2D plane stress, it is possible to prove that the maximum tensile stress is located at the center of the compact and is given by:

$\sigma=\frac{2 F}{\pi D h}$

where $\sigma$ is the maximum tensile stress, $\mathrm{F}$ is the applied force and, D and $h$ are respectively the diameter and thickness of the cylinder. Thus, considering a failure criteria based on the maximum principal stress, the tensile strength is calculated by substituting, in Eq. (1), the force that caused the failure.

The main problem of this test geometry is the contact in the loading area. The contact area between the platens and the cylinder is very small, and it promotes the development of high stresses. If the contact is too small, the failure could be caused by shear effects in this area, leading thus to incorrect failure pattern. 
To avoid this problem, several authors have recommended the use of loading strips that are positioned between the platens and the sample (Li and Wong, 2013).This makes it possible to increase the contact area avoiding shear or compressive failure. Other techniques to increase the contact area have been proposed like the use of curved platens (ISRM, 1978) or the use of flattened discs instead of cylinder (Wang et al., 2004). This last solution, will be further developed below in the article.

In the pharmaceutical field, the problem of contact was studied since the test was introduced. In the article which is the reference for the use of the diametral compression for tablets, Fell and Newton (1970) studied the influence of inserting sheets of blotting paper between the platens and the tablet. Diametral compression tests with and without blotting papers were carried out for five different lactose batches (one crystalline, and four spray-dried noted A-D). For the samples B-D they found that, without blotting paper, the failure was due to compression and shear at the contact with platens. For this case, the use of blotting paper was mandatory to obtain a tensile failure. For the case of crystalline and spray dried A samples, the results were different. For these two products, with and without blotting paper, the failure occurred in tension, i.e. no failure at the contact point was observed. Nevertheless, the values obtained in the two cases were different. They then stated "this raises the question as to which of the values represent the tensile strength of the tablet". The contact problem was also studied more recently by Davies et al. (2007). In their study, they used two contact configurations. The first one was classical flat platens and for the second configuration, they attached semi-circular steel rods to the platens. As it is proved in the article, this last configuration made it possible to have a smaller contact width. In all the cases, they observed failure in tension, but they also observed that for smaller a contact width, they obtained a lower tensile strength by using Eq. (1) which is exactly the same trend obtained by Fell and Newton (1970). Again, due to the failure pattern observed, shear or compression failure at the contact point could not be taken as responsible of the difference in tensile strength obtained for different contact widths. And the question mentioned above about which one of the value is the correct value of the tensile strength has still, to our knowledge, no real answer in the literature.

Nevertheless, the problem of contact is generally discarded in the pharmaceutical field. A great number of studies have been published about the failure of pharmaceutical compacts using the diametral compression test, studying for example the effects of the shape of the compact (Pitt et al., 1988; Podczeck et al., 2013, 2014; Razavi et al., 2015; Shang et al., 2013a, 2013b) or on the effect of the material properties (Procopio et al., 2003). But in nearly all the studies, flat platens are used. The regulatory texts also advise for the use of flat platens (EUP, 2016; USP, 2013) and the value of the diametral compression test is taken as the value of the tensile strength and used, for example, as a material characteristic to calibrate numerical models (Brewin, 2008; Cunningham et al., 2004; Wu et al., 2005).

Another interesting point is that the theoretical development that leads to Eq. (1) is a 2D one. It thus supposes that there is no 3D effects. Nevertheless, 3D effects were demonstrated by both analytical developments (Wijk, 1978) and numerical simulation using the Finite Element Method (FEM) (Ehrnford, 1981; Li and Wong, 2013). All these studies show that, if the contact surface between the cylinder and the platen is too small, high tensile stresses develop on the outer surface of the compact away from the central axis. The value and position of these stresses depend on the contact area, on the thickness of the compact and also on Poisson's ratio. The quantitative determination of these stresses is thus complicated, but as stated by Ehrnford a long time ago, due to these stresses, "peripherical fracture initiation must be regarded as a possibility" (Ehrnford, 1981). Surprisingly, the presence of the maximum tensile stress away from the center is generally not considered and studies about 3D effects in the diametral compression test mainly focus on the variation of the tensile stress at the center along with the thickness of the compact (Podczeck et al., 2013; Yu et al., 2006).

After this brief review, it is clear that the diametral compression test is still not fully understood and that the results obtained (i.e. tensile strength) must be taken with caution if they are to be used as material constants. It is also clear that the problem comes from the contact area between the platen and the compact. Among the solutions to increase this contact, the flattened Brazilian disc was proposed for rocks. It consists in introducing two flat ends to the disc (Wang et al., 2004). This technique has the advantage of introducing a well-defined and quantified contact area between the platens and the tablet. By choosing the correct contact surface, it should thus be possible to suppress the problem of shear failure and also the peripheral tensile stresses due to 3D effects. In the case of pharmaceutical tablets, by designing tools with the proper shape, it is easy to produce flattened tablets.

In this work, we wanted to study, in the case of pharmaceutical tablets, the effect of using the flattened Brazilian disc on the value of the tensile strength obtained by diametral compression. In a first part, FEM studies were performed to show that the use of specially designed punches to obtain flattened compact did not have an impact on the stress and density distributions inside the compact. This part was necessary to prove that the flattened and round tablet had the same density distribution and that their failure behavior could thus be compared. In a second part, the stress and strain distributions inside the compact during the diametral compression were evaluated numerically (FEM). FEM simulations were compared to the results already published in the literature. Then, Digital Image Correlation (DIC) was used to compare the experimental strain fields to the ones obtained numerically in order to prove that FEM simulations gave a good representation of the actual behavior of the compact. Afterwards, the diametral test was performed for both normal and flattened disc geometry to compare the values obtained for the tensile strength. Finally, a high speed camera was used to locate the crack initiation during the test on both geometries.

\section{Material and method}

\subsection{Powders}

Five different powders were used to produce compacts: anhydrous calcium phosphate (aCP) (Anhydrous Emcompress ${ }^{\circledR}$, JRS Pharma, Rosenberg, Germany), calcium phosphate dihydrate (DCP) (Emcompress Premium ${ }^{\circledR}$, JRS Pharma, Rosenberg, Germany), spray-dried lactose monohydrate (SDLac) (Flowlac ${ }^{\circledR}$ 90, Meggle, Wasserburg, Germany), granulated lactose monohydrate (GLac) (Tablettose ${ }^{\circledR} 80$, Meggle, Wasserburg, Germany) and spray-dried mannitol (SDMan) (Pearlitol ${ }^{\circledR}$ 200SD, Roquette, Lestrem, France). To perform the compaction experiments, the products were mixed with $1 \%(\mathrm{w} / \mathrm{w})$ of magnesium stearate (Cooper, Melun, France) to minimize the frictions in the die. The blending was performed at $50 \mathrm{rpm}$ for $5 \mathrm{~min}$ using a turbula mixer (Type T2C, Willy A Bachofen, Muttenz, Switzerland).

\subsection{Compression}

All the compacts were produced using a compaction simulator Stylcam ${ }^{\circledR}$ (Medelpharm, Bourg-en-Bresse, France). This tableting press is a single station press. It is equipped with force sensor (accuracy $10 \mathrm{~N}$ ) and the displacements of the punches are monitored with an accuracy of $0.01 \mathrm{~mm}$. Two different sets of flat-faced euro B punches were used (ACM, Avilly-Saint-Leonard, 
France). The projection of the active surface of the two sets can be found in Fig. 1. The first set was round with a diameter of $11 \mathrm{~mm}$ and made it possible to obtain round tablets (Fig. 1a). The second set was made of punches especially designed to obtain flattened discs (Fig. 1b). All the compacts were obtained using the direct cam mode at a speed of 10 compacts per minutes (total compression time of about $100 \mathrm{~ms}$ ) and under four or five different pressure levels to obtain several densities. To avoid any effect due to the thickness of the compacts, all the compacts manufactured had similar thicknesses around $3.8 \mathrm{~mm}$. The density was calculated using the weight and dimensions of the compacts. A SmarTest 50 semi-automatic tablet testing system (Sotax, Saint-Louis, France) was used to weight and measure the tablets. For the flattened geometry, the distance between the two flat edges was measured with a Mitutoyo micrometer (Kawasaki, Japan).

\subsection{Mechanical characterization}

The diametral compression test was performed using a TA. HDplus texture analyzer (Stable microsystems, Surrey, United Kingdom). Compacts were compressed between two flat surfaces at a constant speed of $0.35 \mathrm{~mm} \mathrm{~s}^{-1}$ with an acquisition frequency of $500 \mathrm{~Hz}$. For each density level of each product, ten compacts were broken. For the high speed video experiments, due to practical reasons, the test was performed on a Zwick Roell Z250 (Zwick Roell, Ulm, Germany) testing machine at the same speed.

\subsection{Digital image correlation}

Digital Image Correlation (DIC) is an optical-based non-contact method used to calculate the $2 \mathrm{D}$ or $3 \mathrm{D}$ full-field surface displacement response of structures to mechanical loading. It is based on pattern matching between two images of the same specimen in two different loading stages (Sutton et al., 2009).

Prior to the tests, the surface of the specimens has been speckled to present a random intensity pattern. This pattern was obtained by applying black ink on the surface of the compact. Image acquisition was made using a camera FASTCAM-APX RS (Photron, San Diego, USA) with a $60 \mathrm{~Hz}$ frequency in order to have sufficient resolution for DIC calculation.

Digital Image Correlation (VIC-2D Vic-Snap 2009, Correlated Solutions Inc., Columbia, SC) was performed using a 70-pixel correlation window size and a 7-pixel step size between two consecutive windows in order to minimize calculation uncertainties. Displacement field and engineering strains have been determined using DIC.

\subsection{FEM simulation}

The FEM modeling was performed using Abaqus ${ }^{\circledR}$ Standard software 6.13 (Dassault Systèmes, Vélizy-Villacoublay, France).

For the studies about the density distribution inside the compact after compression, the mechanical behavior of the
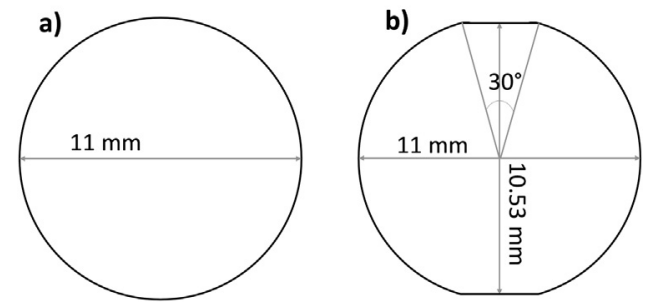

Fig. 1. Projection of the active surface of the punches for the standard (a) and flattened (b) geometries. powder was modeled using Drucker-Prager-Cap model as generally performed in the literature (Brewin, 2008; Cunningham et al., 2004; Wu et al., 2005). The parameters used were characteristic of anhydrous calcium phosphate and were taken from a previous publication (Diarra et al., 2015). For the tooling, the elastic properties of steel were considered $(\mathrm{E}=200 \mathrm{GPa}$ and $\nu=0.3)$. A symmetrical compression was considered (both punch moving at the same time) as it is the case for the tableting machine used in this study. For the example shown in the article, the movement of the punches was monitored to obtain a compaction pressure of about $150 \mathrm{MPa}$ in the axial direction. Using symmetry reasons, only a quarter of the whole geometry was modeled.

For the simulation of the diametral compression, the compact was considered as an elastic material. The value of Young's modulus and Poisson's ratio for the simulation were chosen depending on the compact and will be given in the text. Their determination was done as described elsewhere (Mazel et al., 2012). The platens were also taken as elastic with elastic constants equal to those of steel ( $E=200 \mathrm{GPa}$ and $\nu=0.3$ ). The stress was applied by moving down the platen. Using the symmetry of the system, only an eighth of the compact was modeled (Podczeck et al., 2013).

\subsection{High-speed video}

High-speed video acquisition was performed during diametral compression test using a FASTCAM SA5 (Photron, San Diego, USA). The frame rate was 372000 images per second. We used for standard tablets a frame size of $320 \times 40$ pixels and for flattened tablet a frame size of $256 \times 48$ pixels. The frame was centered on the tablet. To enhance the visualization, green ink was applied on the surface. This color corresponds to the best spectral response of the camera.

\section{Results and discussion}

\subsection{Density distribution inside the compacts}

The first concern was to verify that the modification of the tooling to obtain flattened tablets did not have important consequences concerning the stress and density distributions inside the tablet. For example, it is known that changing the curvature of the punch has dramatic consequences on the density distribution inside the tablets (Diarra et al., 2015; Sinka et al., 2004).

Nevertheless, in our case, the modification did not concern the curvature of the punches. The used punches were flat in both cases. The only modification was that, in the case of the punches for the flattened geometry, the cylindrical shape was slightly modified to introduce two flat ends. As these flat ends were parallel to the direction of compression, not real influence was expected on the stress and density distributions inside the tablet.

To confirm this fact, numerical simulation were performed for both geometries. As mentioned above, the parameters taken for the simulation were those of aCP. As an example, a symmetrical compression up to a pressure of $150 \mathrm{MPa}$ was modelled. The results can be seen in Fig. 2. We first checked that the radial stress was not influenced by the presence of the flat part. As it can be seen in Fig. $2 \mathrm{a}$ and b, the radial stress normal to the flat part ( $\sigma_{\mathrm{yy}}$ Fig. 2a) and the radial stress in the perpendicular direction ( $\sigma_{\mathrm{xx}}$ Fig. $\left.2 \mathrm{~b}\right)$ are comparable and, at the center, the difference is less than $1 \%$ (i.e. the radial stress is nearly isotropic). The two density distributions are also completely comparable and only small differences were observed near the end of the flat portion of the crown (Fig. $2 \mathrm{c}$ and d). This means that the compacts of both geometries should be similar in terms of mechanical properties and of mechanical 

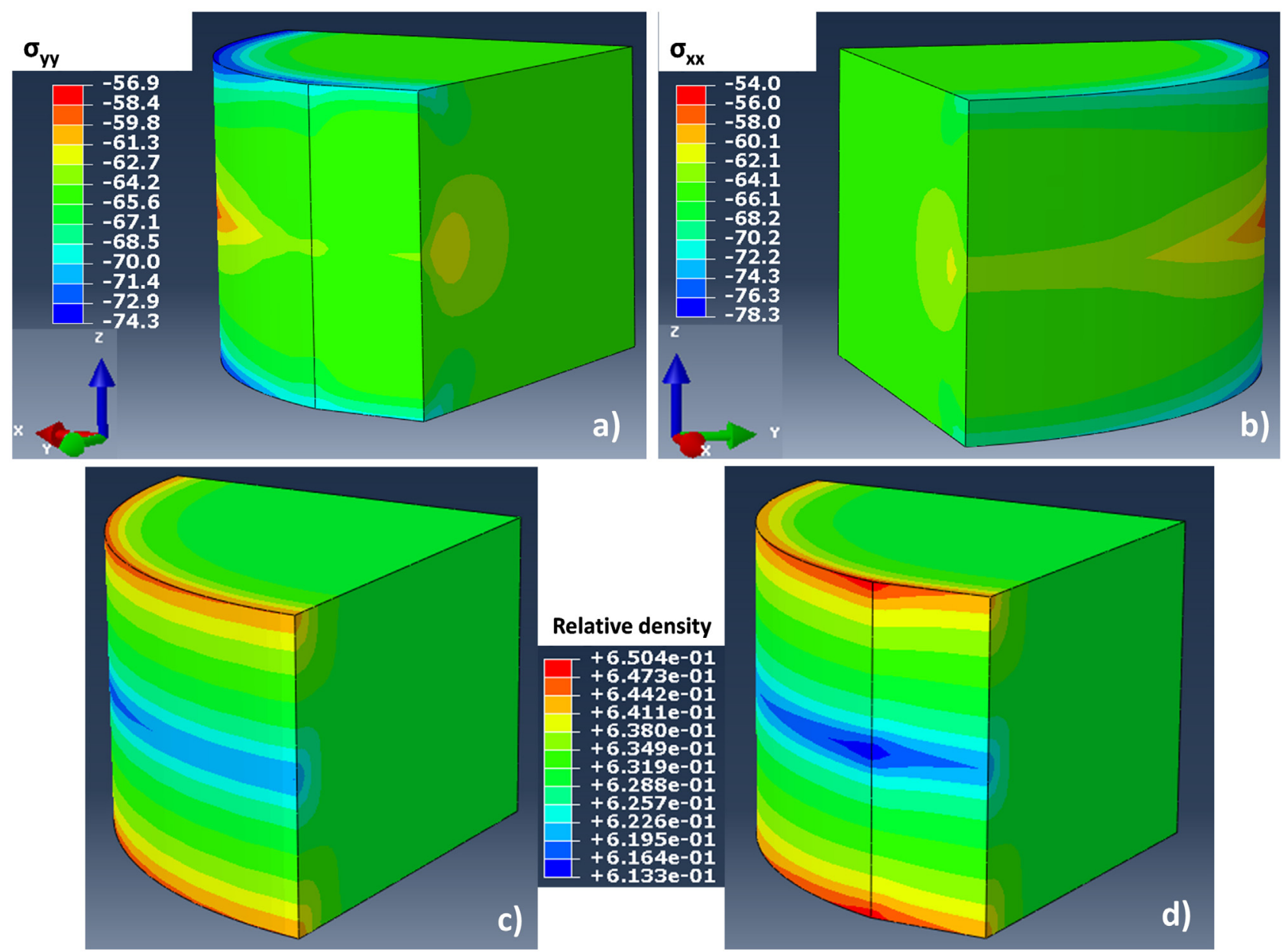

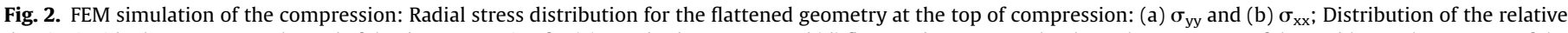

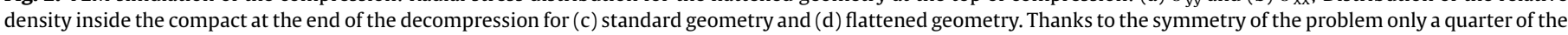
geometry is represented.

strength. The tensile strength of the two geometries should be identical. It should thus be possible to compare the results obtained during diametral compression for both geometries.

\subsection{FEM simulation of the diametral compression test}

The second step of this study was to understand the consequence of flattening a compact on the stress and strain distributions during the diametral test by using FEM simulation. As an example, we chose the elastic properties of a compact made of SDLac obtained under a pressure of $100 \mathrm{MPa}$, which corresponds to a porosity level of $19 \%$. The elastic constants for the simulation were determined as described elsewhere (Mazel et al., 2012). The results gave a Young's modulus of $4.2 \mathrm{GPa}$ and a Poisson's ratio of 0.25 . The thickness was set to $3.8 \mathrm{~mm}$ and the diameter to $11.03 \mathrm{~mm}$ to match experimental compacts that were produced and that were used for the following section. The results of the simulations are presented in Fig. 3. The displacement was set to obtain an applied force of $100 \mathrm{~N}$ on the compact.

As already described above, only an eighth of the compact is represented. In Fig. 3, the side of the compact in front of the picture (direction $\mathrm{z}$ positive) is the surface of the compact and the side on the back (direction $z$ negative) is the central plane. The figure represents the normal stress and strain in the $\mathrm{x}$ direction (i.e. $\sigma_{\mathrm{xx}}$ and $\varepsilon_{\mathrm{xx}}$ ). To make the representation as clear as possible we only represented the tensile values (i.e. positive). The part of the tablet where $\sigma_{\mathrm{xx}}$ and $\varepsilon_{\mathrm{xx}}$ are compressive appears in black.
The results obtained on the standard geometry (Fig. 3a and b) are comparable to those presented by Li and Wong (2013). The highest tensile strains are located slightly under the contact point. For the tensile stresses, there is a clear 3D effect as already mentioned in other publications (Ehrnford, 1981; Li and Wong, 2013). The maximum tensile stress is located on the surface of the compact and is not centered. The obtained value (i.e. $\sigma_{\mathrm{xx}}=3.7 \mathrm{MPa}$ ) is moreover superior to the one obtained using Eq. (1) which would be in this case equal to $1.5 \mathrm{MPa}$. To illustrate this fact, Fig. 4 represents the same situation than Fig. $3 \mathrm{~b}$ but the color scale was modified in order to have, in color, the part of the compact where the tensile stress is superior to the one obtained using Eq. (1). It can be seen that an important part of the compact is submitted to a higher tensile stress.

To conclude on the standard geometry, neither the highest tensile strain nor the highest tensile stress is located at the center of the compact. As concluded by Ehrnford (Ehrnford, 1981), a fracture initiation away from the center of the compact must be regarded as a possibility. And in this case, the use of Eq. (1) would lead to an under estimation of the tensile strength of the compact.

The case of the flattened disc is presented in Fig. $3 \mathrm{c}$ and $\mathrm{d}$. The stress and strain distributions are completely different from those obtained in the standard geometry. Both maximum stresses and strains are now located on the central axis of the compact. The values on the surface are slightly higher than those obtained in the central plan. The maximum tensile strain $\left(\varepsilon_{\mathrm{xx}}=5.69 .10^{-4}\right)$ obtained for the flattened geometry is much lower than for the standard one 

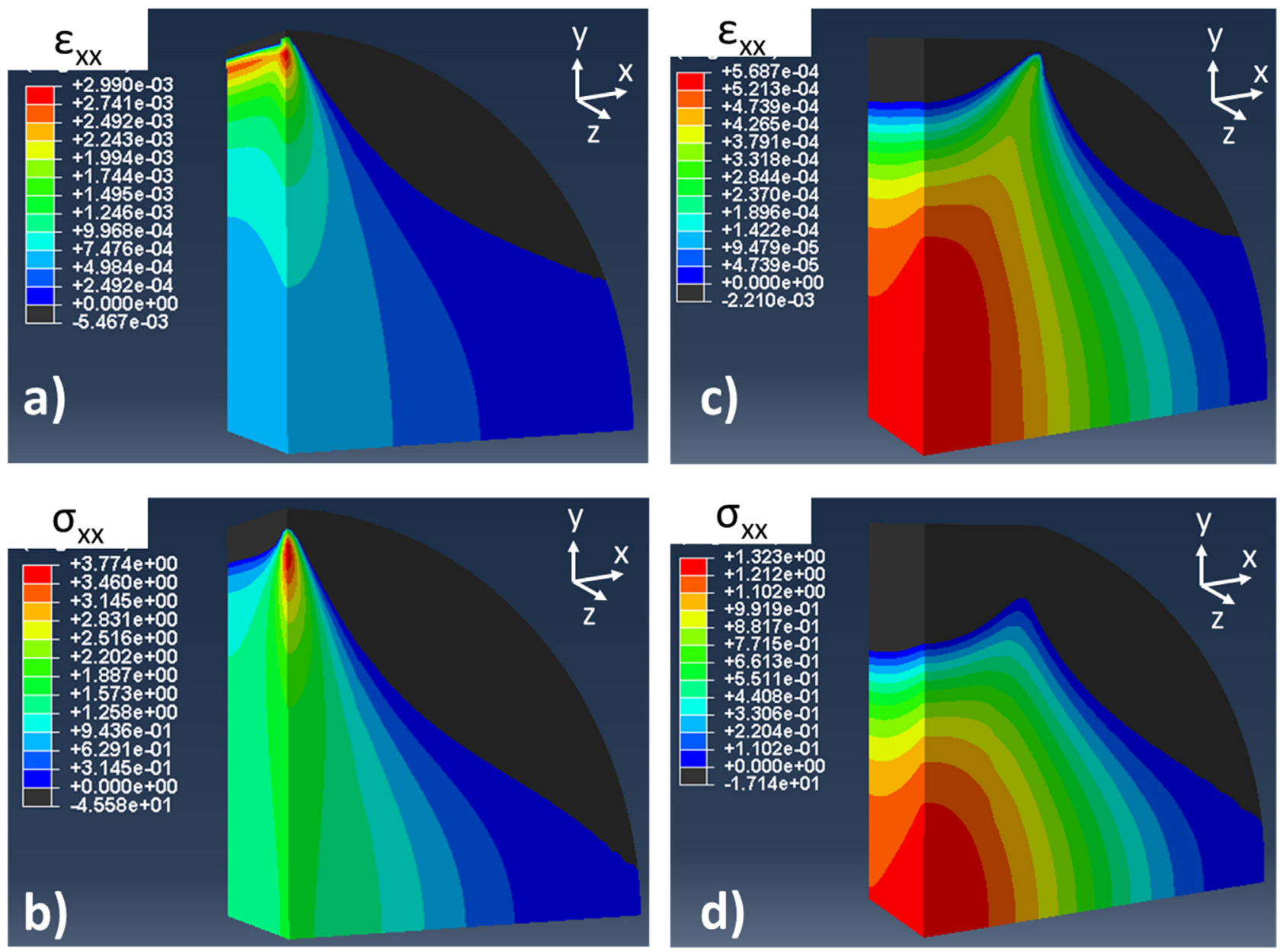

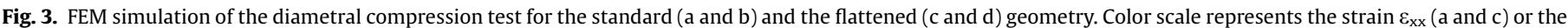
stress $\sigma_{\mathrm{xx}}$ (b and d) (in MPa).

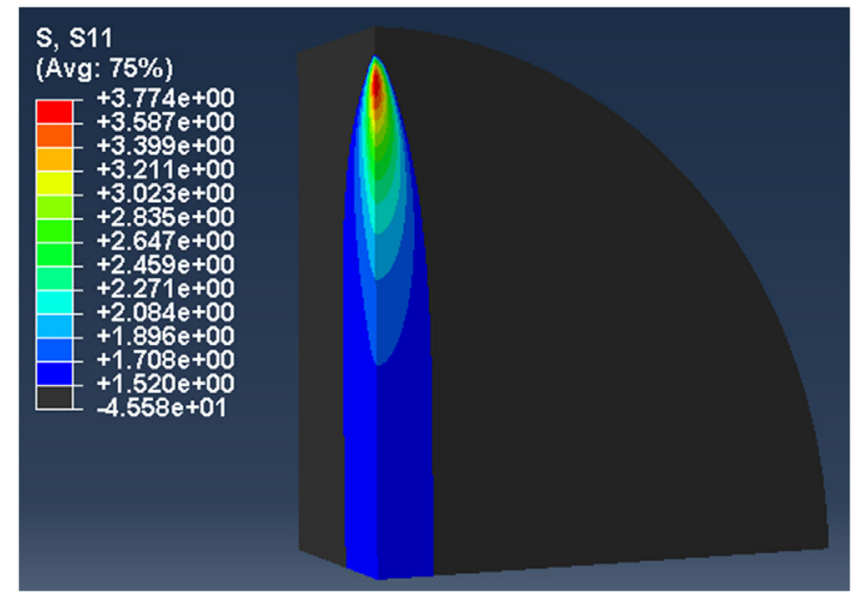

Fig. 4. FEM simulation of the diametral compression for the standard geometry. The color scale represents $\sigma_{\mathrm{xx}}$ and has been limited to the values that exceed the value given by Eq. (1).

$\left(\varepsilon_{\mathrm{xx}}=2.99 .10^{-3}\right)$. In the case of the maximum tensile stress, the value is slightly lower than the one predicted by Eq. (1) $\left(\sigma_{\mathrm{xx}}=1.32 \mathrm{MPa}\right)$. This was expected as several publications already shown that, increasing the contact area on which the load is applied, promotes a decrease of the tensile stress at the center (Hondros, 1959). Thus, to obtain the right value of the tensile stress, a correction factor must be added to Eq. (1). In the case of the flattened disc with a contact angle of $30^{\circ}$, Wang et al. (2004) recommended a correction factor of 0.92 . Nevertheless, their study was based on 2D calculations. We thus preferred to recalculate the correction factors by using 3D FEM simulations. The geometry used was the one presented in Fig. 3 and we used load up to $500 \mathrm{~N}$ which is the highest value that can be obtained experimentally on our device. We found that the correction factor was not influenced by the load value. Only small changes were noticed when changing Young's modulus and Poisson's ratio as expected considering the theory of linear elasticity (Podczeck et al., 2013; Procopio et al., 2003). No significant variation were found for the different thicknesses used in this study (between 3.6 and $4 \mathrm{~mm}$ ). For all the cases a constant correction factor of 0.87 was thus used. Moreover, the ratio between the compressive stress and the tensile stress at the location of the maximal tensile stress was also checked. A value of 3.2 was found which is close to the value of 3 that is found theoretically for the standard geometry at the center (Procopio et al., 2003). The difference been small (less than 10\%), it is thus expected that the compressive stress will play the same role in both geometries and, as a consequence, could not cause a change in the tensile strength value.

Finally, the simulations presented in Fig. 3 indicate that the use of a flattened disc is favorable to obtain a crack initiation at the center of the compact as, at this location, both stresses and strains are maximum. On the contrary, for the case of the standard geometry, neither the maximum tensile stress nor the maximum 
tensile strain is located at the center. The possibility of a fracture initiation away from the center of the compact must be regarded as a possibility.

Nevertheless, all the developments made on the diametral test were based on the assumption of an elastic behavior of the compact during the diametral compression test. In the next part, the simulation results were compared to experimental ones to study if the elastic behavior is a good representation of the actual behavior of the compact during the test.

\subsection{Comparison with experimental results (DIC)}

DIC was used to study the effective mechanical behavior of the compact during the test. This technique makes it possible, as mentioned before, to measure the displacement fields at the surface of the compact during the test. The displacement can then be converted in strain field. The idea was to compare these experimental results to those obtained using FEM simulation considering a linear elastic behavior.

As an example, the case of compact of SDLac obtained under an axial pressure of $100 \mathrm{MPa}$ was again considered. The choice of this product was based on the fact that, as it will be demonstrated below, SDLac makes it possible to obtain strong compacts. During the diametral compression test, relatively high forces can thus be applied before failure, which makes it possible to obtained relatively high strains compared to other products like aCP for example. This product is thus favorable to obtain reliable results using DIC technique.

The exact compact geometry was implemented for the FEM simulation and the applied force was equal to the one applied experimentally. Fig. 5 presents both experimental (DIC) and numerical (FEM) strain fields in the X-direction $\left(\varepsilon_{\mathrm{xx}}\right)$. To make the comparison easier, the whole surface of the compact was represented for the FEM results by using the symmetries of the problem. Moreover, the scale was also slightly modified to enhanced the contrast (i.e. some areas were excluded). The results of FEM are directly comparable to those presented in Fig. 3 but the applied force is slightly different to match the experimental one and to represent the compact just before failure.

The first comment is that from a qualitative point of view, the strain distributions obtained with DIC were comparable to the one predicted using FEM. These results are also coherent with the existing literature (Stirling et al., 2013). As mentioned above, for the standard geometry, the maximum tensile strain was located away from the center. On the contrary, for the flattened geometry the maximum tensile strain is located at the center. The comparison of the patterns obtained by DIC and FEM makes it possible to conclude that the mechanical behavior of the compact during the test is, at least qualitatively, correctly represented by a linear elastic model.

It is also interesting to look at the result from a quantitative point of view. As it can be seen on Fig. $5 \mathrm{a}$ and $\mathrm{c}$, the strains measured are between $10^{-4}$ and $10^{-3}$. This value is quite small and corresponds to the limit of validity of DIC measurements. Nevertheless by comparing DIC and FEM, it is clear that the quantitative values are in the same order of magnitude. For example in the flattened geometry, the maximum tensile strain value in both cases is around $10^{-3}$ and for the standard geometry the strain value at the center is around $7 \cdot 10^{-4}$. The value of the elastic moduli that were used for the simulation are thus in the good order of magnitude.

These results demonstrate that, for this product, the linear elastic model is suitable to describe the mechanical behavior of the compact during the test and the value of the elastic moduli used are in the good order of magnitude. As a consequence, the calculated strain and stress fields calculated by FEM can be used to interpret the failure using the diametral compression test.
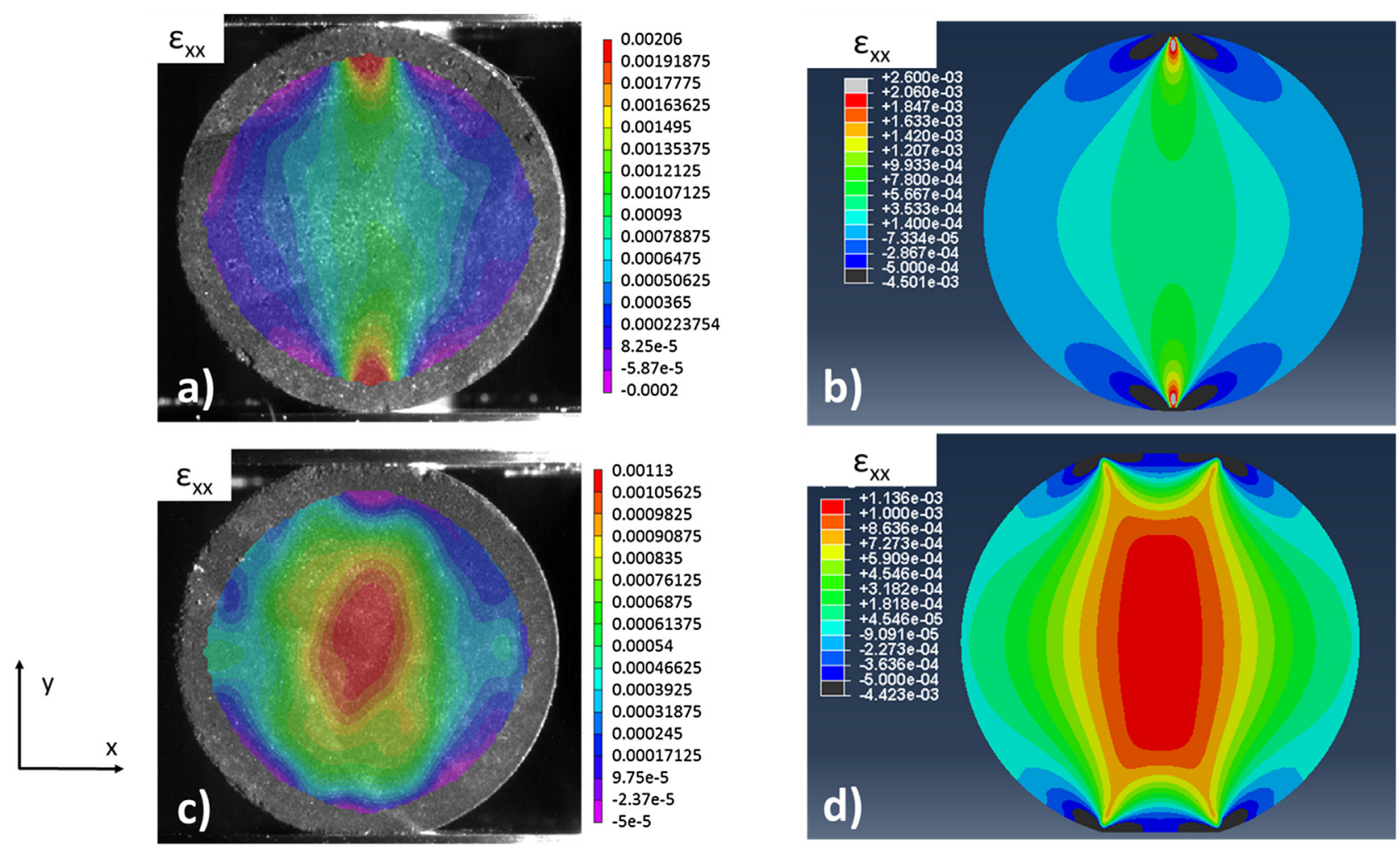

Fig. 5. Comparison between the strain fields $\left(\varepsilon_{\mathrm{xx}}\right)$ obtained using DIC ( $\mathrm{a}$ and c) and FEM (b and d) for the standard (a and b) and the flattened (c and d) geometries. 


\subsection{Failure results}

For the five products considered in this study, compacts were made under at least four pressure levels in order to obtain compacts with increasing mechanical strength. Both standard and flattened compacts were manufactured under these different loads. For each product and density, ten compacts were, afterwards, measured and broken diametrically as described above. For each failure test, the tensile strength was calculated. For the standard geometry, Eq. (1) was used and for the flattened geometry, Eq. (1) was multiplied by 0.87 to take into account the thickness effect as explained in Section 3.2. To quantify the difference between the values obtained in both geometries, a tensile strength difference was calculated by using the tensile strength for the standard geometry $\left(\sigma_{s}\right)$ and the tensile strength for the flattened geometry $\left(\sigma_{\mathrm{f}}\right)$. The following expression was used:

Tensile strength difference $=\frac{\sigma_{f}-\sigma_{s}}{\sigma_{s}} \times 100$

The results are presented in Fig. 6. To facilitate the visualization of the results, products are presented in two groups (Fig. 6a and b). On each graph, the $\mathrm{X}$-axis is the tensile strength obtained with the standard geometry and the Y-axis is the one obtained for the flattened compacts. It was found that for each product the points align nicely on a straight line with an intercept equal to zero (For all the products $R^{2}$ is superior to 0.99 ). The slope of each straight line can be seen in Fig. 6. This leads to two comments.

The first comment is that in all the cases, the slope of the line is superior to 1 . This means that the value obtained for the standard geometry is lower than the one obtained for the flattened geometry. As already mentioned in the literature, the standard geometry underestimates the tensile strength.

The second interesting point is the fact that the points are aligned. It means that for one product, whatever the strength, the tensile strength difference is constant. The tensile strength difference can be calculated directly from the slope. The results are presented in Table 1.

The tensile strength difference ranged from $17 \%$ to $71 \%$ depending on the product. But it is worth noting that for all the products, the failure pattern using the diametral test indicated a failure in tension. It was not possible to infer the differences between the products only by looking at the failure pattern. This means that, if it is intended to produce a compact with a tensile strength of $2 \mathrm{MPa}$ as recommended in the literature (Sun et al., 2009) and if this tensile strength is measured using the standard geometry, it could correspond, in the case of the products of this study, to a tensile strength measured using the flattened geometry ranging from 2.34 to $3.44 \mathrm{MPa}$. These values correspond, in reality, to very different compact strengths, and the test with the standard geometry is unable to discriminate them. This result is important for development studies. Using the standard geometry, the use of a
Table 1

Tensile strength difference for the different products.

\begin{tabular}{ll}
\hline Product & Tensile strength difference \\
\hline aCP & $17 \%$ \\
DCP & $29 \%$ \\
SDMan & $34 \%$ \\
GLac & $35 \%$ \\
SDLac & $71 \%$ \\
\hline
\end{tabular}

specification of a tensile strength of $2 \mathrm{MPa}$ for the development of a new compact is questionable as, depending on the product, it can in fact correspond to actual very different mechanical strength.

The underestimation of the tensile strength using the standard geometry was already predicted above, considering the FEM results. As the maximum tensile stress is not at the center, a failure away for the center could occur. If it was the case, calculating the tensile strength using Eq. (1) which considers the stress at the center would lead to an underestimation of the actual tensile strength. So all the results are consistent with the fact that, for the standard geometry, the failure occurs away from the center. In order to confirm this fact, high-speed video was used to locate the crack initiation in the compact during the test for both geometry.

\subsection{High-speed video results}

Filmed diametral compression tests with high speed video camera were performed. For practical reason, it was not possible to film experiment with all the compacts presented above. So we chose, for each product, compacts that corresponded to an apparent tensile strength of $2 \mathrm{MPa}$ using the standard geometry. At least four compacts for each geometry were broken and the video was then analyzed to detect the crack initiation. For SDLac and Glac, it was possible for both geometry to detect correctly the crack initiation. For aCP and DCP, the resolution of the camera at the frame speed used was not enough to be able to locate the initiation properly, especially in the case of the flattened geometry. For SDMan, we found that in the standard geometry, the crack tip velocity was very high (several hundred $\mathrm{m} / \mathrm{s}$ ) and that our set-up (frame rate/resolution balance) did not make it possible to locate properly the initiation.

Fig. 7 presents representative examples of crack initiation and propagation for SDLac and GLac for both geometries. For the standard geometry the failure initiates away from the center in the direction of one of the platen before propagating through the whole sample. On the contrary for the flattened geometry, the failure is located at the center at the same distance from each platen.

The videos confirm all the results presented above. The crack initiation is away from the center for the standard geometry as predicted by FEM simulation. On the contrary, for the flattened
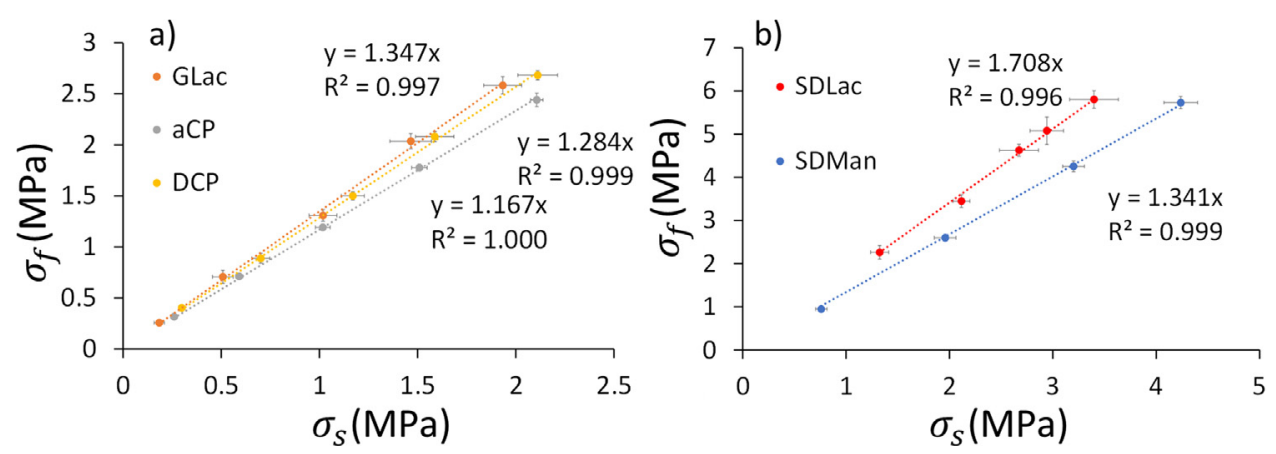

Fig. 6. Tensile strength for the flattened geometry $\left(\sigma_{\mathrm{f}}\right)$ as a function of the tensile strength for the standard geometry $\left(\sigma_{\mathrm{s}}\right)$ for the different products. 


\section{GLac}

SDLac

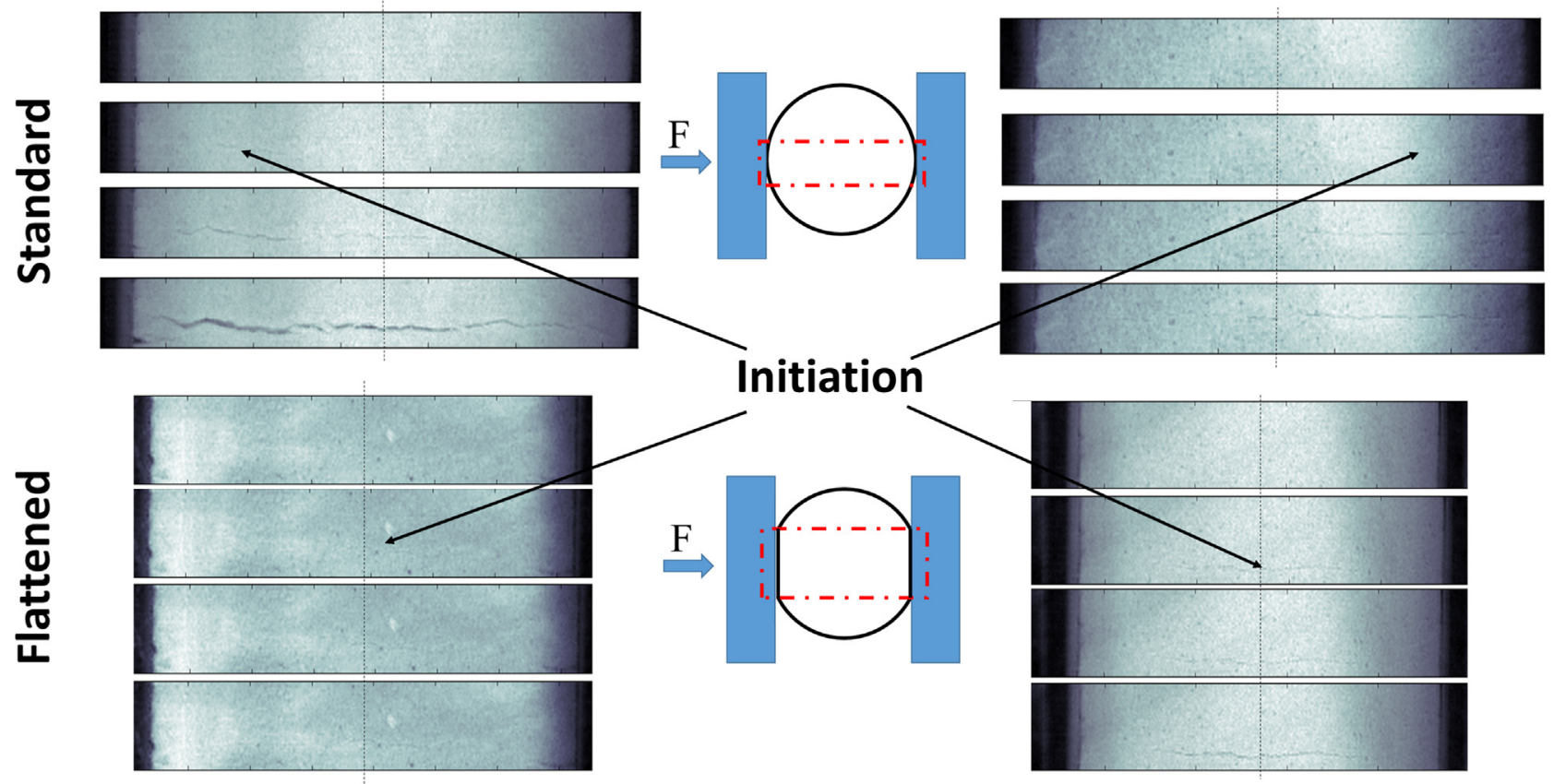

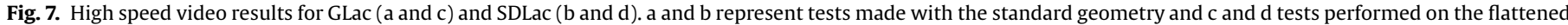
geometry. On each serie of photos, time evolution goes from the top to the bottom.

geometry, the failure starts at the center were the stress and strain are maximum. We can thus conclude that the explanation of the underestimation of the tensile strength using the standard geometry is caused by the fact that the failure does not initiate at the center of the compact. Using the standard geometry to perform the diametral compression of pharmaceutical tablet does thus not make it possible to calculate correctly the tensile strength of the tablet.

\section{Conclusion}

FEM analysis made it possible to emphasize that, when performing the diametral test using the standard geometry, neither the maximum tensile strain nor the maximum tensile stress is located at the center of the compact. Out of center crack initiation must thus be considered as a possibility. On the contrary, when the flattened geometry is used both the maximum tensile stress and strain are located at the center. This geometry is thus more favorable to measure the tensile strength.

By using DIC technique, it was possible to compare the strain fields obtained by simulation to those obtained experimentally. They were found comparable which means that the linear elastic model used in the simulation is suitable to represent the mechanical behavior of the compact during the tests and that the stress fields calculated by FEM are usable to interpret the test.

Experimental breaking tests using both geometries indicate that the standard geometry always underestimates the tensile strength of the compact. Moreover for one product, the tensile strength difference is not dependent on the strength of the compact. Nevertheless the tensile strength difference is dependent on the product. This means that two compacts which have the same tensile strength as measured with the standard geometry, could have in fact very different mechanical strengths.

Finally, high speed video experiments made it possible to localize the crack initiation during the tests. It confirmed what was foreseen in the simulations: for the standard geometry, the crack initiation is away from the center whereas centered fracture is obtained for the flattened technology. This explains why the standard geometry always underestimates the tensile strength.

As we mentioned in the introduction, in 1970, Fell and Newton asked the question "which of the values represent the tensile strength of the tablet" (Fell and Newton, 1970). All the results presented in the paper make it possible to answer that the value obtained using the diametral test on the standard geometry does not correspond to the tensile strength of the tablet, and should thus not be used as such.

\section{Appendix A. Supplementary data}

Supplementary data associated with this article can be found, in the online version, at http://dx.doi.org/10.1016/j. ijpharm.2016.09.088.

\section{References}

Brewin, P.R., 2008. Modelling of Powder Die Compaction. Springer, London.

Carneiro, F., 1943. A new method to determine the tensile strength of concrete. Proc. 5th Meet. Braz. Assoc. Tech. Rules 126-129.

Cunningham, J.C., Sinka, I.C., Zavaliangos, A., 2004. Analysis of tablet compaction. I. Characterization of mechanical behavior of powder and powder/tooling friction. J. Pharm. Sci. 93, 2022-2039.

Davies, P.N., Worthington, H.E.C., Podczeck, F., Newton, J.M., 2007. The determination of the mechanical strength of tablets of different shapes. Eur. J. Pharm. Biopharm. 67, 268-276.

Diarra, H., Mazel, V., Busignies, V., Tchoreloff, P., 2015. Investigating the effect of tablet thickness and punch curvature on density distribution using finite elements method. Int. J. Pharm. 493, 121-128.

EUP, 2016. 2.9.8 resistance to crushing of tablets, European Pharmacopeia. Eur Directorate Qual. Med. Healthcare 299.

Ehrnford, L., 1981. Stress distribution in diametral compression tests. Acta Odontol. Scand. 39, 55-60.

Fairbairn, E.M.R., Ulm, F.-J., 2002. A tribute to Fernando L.L.B. Carneiro (1913-2001) engineer and scientist who invented the Brazilian test. Mater. Struct. 35, 195-196.

Fell, J.T., Newton, J.M., 1968. The tensile strength of lactose tablets. J. Pharm. Pharmacol. 20, 657-659. 
Fell, J.T., Newton, J.M., 1970. Determination of tablet strength by the diametralcompression test. J. Pharm. Sci. 59, 688-691.

Hondros, G., 1959. The evaluation of Poisson's ratio and the modulus of materials of a low tensile resistance by the Brazilian (indirect tensile) test with particular reference to concrete. Aust. J. Appl. Sci. 10, 243-268.

ISRM, 1978. Suggested methods for determining tensile strength of rock materials. Int. J. Rock Mech. Min. Sci. Geomech. Abstr. 15, 99-103.

Li, D., Wong, L.N.Y., 2013. The Brazilian disc test for rock mechanics applications: review and new insights. Rock Mech. Rock Eng. 46, 269-287.

Mazel, V., Busignies, V., Diarra, H., Tchoreloff, P., 2012. Measurements of elastic moduli of pharmaceutical compacts: a new methodology using double compaction on a compaction simulator. J. Pharm. Sci. 101, 2220-2228.

Pitt, K.G., Newton, J.M., Stanley, P., 1988. Tensile fracture of doubly-convex cylindrical discs under diametral loading. J. Mater. Sci. 23, 2723-2728.

Podczeck, F., Drake, K.R., Newton, J.M., 2013. Investigations into the tensile failure of doubly-convex cylindrical tablets under diametral loading using finite element methodology. Int. J. Pharm. 454, 412-424.

Podczeck, F., Newton, J.M., Fromme, P., 2014. Theoretical investigations into the influence of the position of a breaking line on the tensile failure of flat, round bevel-edged tablets using finite element methodology (FEM) and its practical relevance for industrial tablet strength testing. Int. J. Pharm. 477, 306-316.

Procopio, A.T., Zavaliangos, A., Cunningham, J.C., 2003. Analysis of the diametrical compression test and the applicability to plastically deforming materials. J. Mater. Sci. 38, 3629-3639.

Razavi, S.M. Gonzalez, M., Cuitiño, A.M., 2015. General and mechanistic optimal relationships for tensile strength of doubly convex tablets under diametrical compression. Int. J. Pharm. 484, 29-37.

Shang, C., Sinka, I.C., Jayaraman, B., Pan, J., 2013a. Break force and tensile strength relationships for curved faced tablets subject to diametrical compression. Int. J Pharm. 442, 57-64.
Shang, C., Sinka, I.C., Pan, J., 2013b. Modelling of the break force of tablets under diametrical compression. Int. J. Pharm. 445, 99-107.

Sinka, I.C., Cunningham, J.C., Zavaliangos, A., 2004. Analysis of tablet compaction. II. Finite element analysis of density distributions in convex tablets. J. Pharm. Sci. 93, 2040-2053.

Stirling, R.A., Simpson, D.J., Davie, C.T., 2013. The application of digital image correlation to Brazilian testing of sandstone. Int. J. Rock Mech. Min. Sci. 60,1-11.

Sun, C.C., Hou, H., Gao, P., Ma, C., Medina, C., Alvarez, F.J., 2009. Development of a high drug load tablet formulation based on assessment of powder manufacturability: moving towards quality by design. J. Pharm. Sci. 98, 239-247.

Sutton, M.A., Orteu, J.-J., Schreier, H.W., 2009. Image Correlation for Shape, Motion and Deformation Measurements: Basic Concepts, Theory and Applications. Springer, New York, N.Y.

USP, 2013. <1217> Tablet Breaking Force, The United States Pharmacopeia-National Formulary, USP35 NF30. U.S. Pharmacopeia.

Wang, Q.Z., Jia, X.M., Kou, S.Q., Zhang, Z.X., Lindqvist, P.A., 2004. The flattened Brazilian disc specimen used for testing elastic modulus, tensile strength and fracture toughness of brittle rocks: analytical and numerical results. Int. J. Rock Mech. Min. Sci. 41, 245-253.

Wijk, G., 1978. Some new theoretical aspects of indirect measurements of the tensile strength of rocks. Int. J. Rock Mech. Min. Sci. Geomech. Abstr. 15, 149-160.

Wu, C.Y., Ruddy, O.M., Bentham, A.C., Hancock, B.C., Best, S.M., Elliott, J.A., 2005. Modelling the mechanical behaviour of pharmaceutical powders during compaction. Powder Technol. 152, 107-117.

Yu, Y., Yin, J., Zhong, Z., 2006. Shape effects in the Brazilian tensile strength test and a 3D FEM correction. Int. J. Rock Mech. Min. Sci. 43, 623-627. 